\title{
System Verification of Hardware Optimization Based on Edge Detection
}

\author{
Xinwei Niu, Jeffrey Fan \\ Department of Electrical and Computer Engineering, Florida International University, Miami, USA \\ Email: Xinwei.Niu@fiu.edu, Jeffrey.Fan@fiu.edu
}

Received March 21, 2013; revised April 21, 2013; accepted April 30, 2013

Copyright (c) 2013 Xinwei Niu, Jeffrey Fan. This is an open access article distributed under the Creative Commons Attribution License, which permits unrestricted use, distribution, and reproduction in any medium, provided the original work is properly cited.

\begin{abstract}
Nowadays, digital camera based remote controllers are widely used in people’s daily lives. It is known that the edge detection process plays an essential role in remote controlled applications. In this paper, a system verification platform of hardware optimization based on the edge detection is proposed. The Field-Programmable Gate Array (FPGA) validation is an important step in the Integrated Circuit (IC) design workflow. The Sobel edge detection algorithm is chosen and optimized through the FPGA verification platform. Hardware optimization techniques are used to create a high performance, low cost design. The Sobel edge detection operator is designed and mounted through the system Advanced High-performance Bus (AHB). Different FPGA boards are used for evaluation purposes. It is proved that with the proposed hardware optimization method, the hardware design of the Sobel edge detection operator can save 6\% of on-chip resources for the Sobel core calculation and $42 \%$ for the whole frame calculation.
\end{abstract}

Keywords: IC; AHB; FPGA; Hardware Optimization; Sobel Edge Detection

\section{Introduction}

The technology evolves rapidly in these years. Currently, people enjoy many high-tech products such as gesture based remote controller by using digital cameras to extract valuable information. It is known that the edge detection plays an important role in the remote control process [1]. The edge detection is used to process the input data and extracts the key feature of the data for further steps. Several edge detection algorithms can be used to identify the edge of one image frame. In this paper, the Sobel edge detection is designed and verified by using the verification platform.

Integrated Circuit (IC) chip manufacture involves a variety of processes. The basic rules are the same even though there may be different kinds of design flows. In the IC design flow, Field-Programmable Gate Array (FPGA) verification is an important step. Benefited from the reconfigurabilities of the FPGA, designers can verify their design at the early stage of the IC design flow. Thus, design defects can be found and eliminated to save design cycles and costs.

The edge detection is the most commonly used approach to detect discontinuities in gray level by far. It is widely used to extract the texture of the item in one picture. There are many edge detection operators based on the gradient detection. In this research, the Sobel operator for the edge detection and a verification platform is used to test the proposed hardware design.

There are several previous researches of the edge detection design using the Sobel operator. S. Halder et al. designed a Sobel operator based on the optimized algorithm in [2]. However, their design used too many dividers and multipliers. Thus, too many on-chip resources are used without significant performance improvement. Besides, they did not have a complete solution for the Sobel edge detection. T. A. Abbasi et al. proposed an FPGAbased architecture for the Sobel edge detection operator [3]. However, they used two random-access memory (RAM) to store the data, one for the original data, and the other for the result data. Thus, the resource consumptions are too high. C. Pradabpet et al. proposed that they could operate the Sobel operation efficiently [4]. However, their design can only run at a very low frequency, so the efficiency is relatively low. Moreover, they still need to use two storage spaces to save the data. Another design based on Sobel operator was described in [5], the author designed a massive pipeline for algorithm calculations. This design can increase the performance significantly. However, the System-on-a-Chip (SoC) may not able to allocate enough on-chip resources for this design. Thus, the design was not a balanced one if implemented into 
SoCs. V. Sanduja and R. Patial designed a complete edge detection system based on the Sobel operator, and their design had an accurate result for each pixel. However, the costs of the resources are still much higher by using two separate memory parts to store the data [6].

The rest of the paper is organized as follows. In Section 2, an overview of the IC design flow and the Sobel edge detection is presented. Section 3 explains the architecture of our hardware verification platform. In Section 4 , the optimized hardware of the Sobel edge detection operator is evaluated, and then the verification platform is demonstrated. Finally, conclusions and future works are drawn in Section 5.

\section{Background}

Designers put different efforts in the process of making a successful IC. As shown in Figure 1, the most important stage in the design flow is the information acquisition stage. Designers do some researches on the aimed design including specifications, algorithms and even the architecture. With a clear understanding of the whole design, designers can go ahead for the next stage.

In the architectural design stage, designers must be familiar with all the related knowledge of the design. The selected algorithm is directly related to the structure, this is why we need to first verify and generate the most suitable algorithm form. The best architecture is the one with the fastest speed, the lowest power consumption and the minimum chip size.

Hardware designers use the hardware description language to write the source code. After that, designers must write the test bench for their design. Test bench provides simulation models for designs.

Then it goes to the Register Transfer Level (RTL) simulation, which is also called behavioral simulation. It is based on the RTL function but not timing consideration. If a designer uses an FPGA to develop the circuit, the design code can be synthesized into the FPGA netlist. The FPGA verification with the real test environment can find most of the problems.

The design code is viewed as a good one once it passes the FPGA verification stage. Then, the code is synthesized into a netlist for the chip layout. After that, designers do simulation again, which is called pre-simulation. On the pre-simulation stage, timing issues are added to the simulation, so the simulation is closer to the performance of the real chip. The timing is only added to the cells and registers, but not wires. If the pre-simulation results are not as expected, the source code should be re-designed.

After the chip layout process, another netlist is generated according to the real wires of the chip. With the consideration of the wire delay, the design goes to the post-simulation stage. If post-simulation results meet the design requirements, the design is ready for the chip fabrication.

The manufactured chip is packaged and mounted in the system to check whether it is good or not. The test pattern should have higher coverage for all the possibilities. The higher the coverage, the better of the yield of the chip. The tester uses the probe card to check the chip on the wafer. When the chip passes the chip probe stage, the wafer can be cut down for packaging. After packaging, the IC chips are tested again to make sure the chips are good. The last step in the entire workflow is to test the IC mounted on the real system.

The edge detection is the most widely used method to detect discontinuities in one image by far. An edge is a

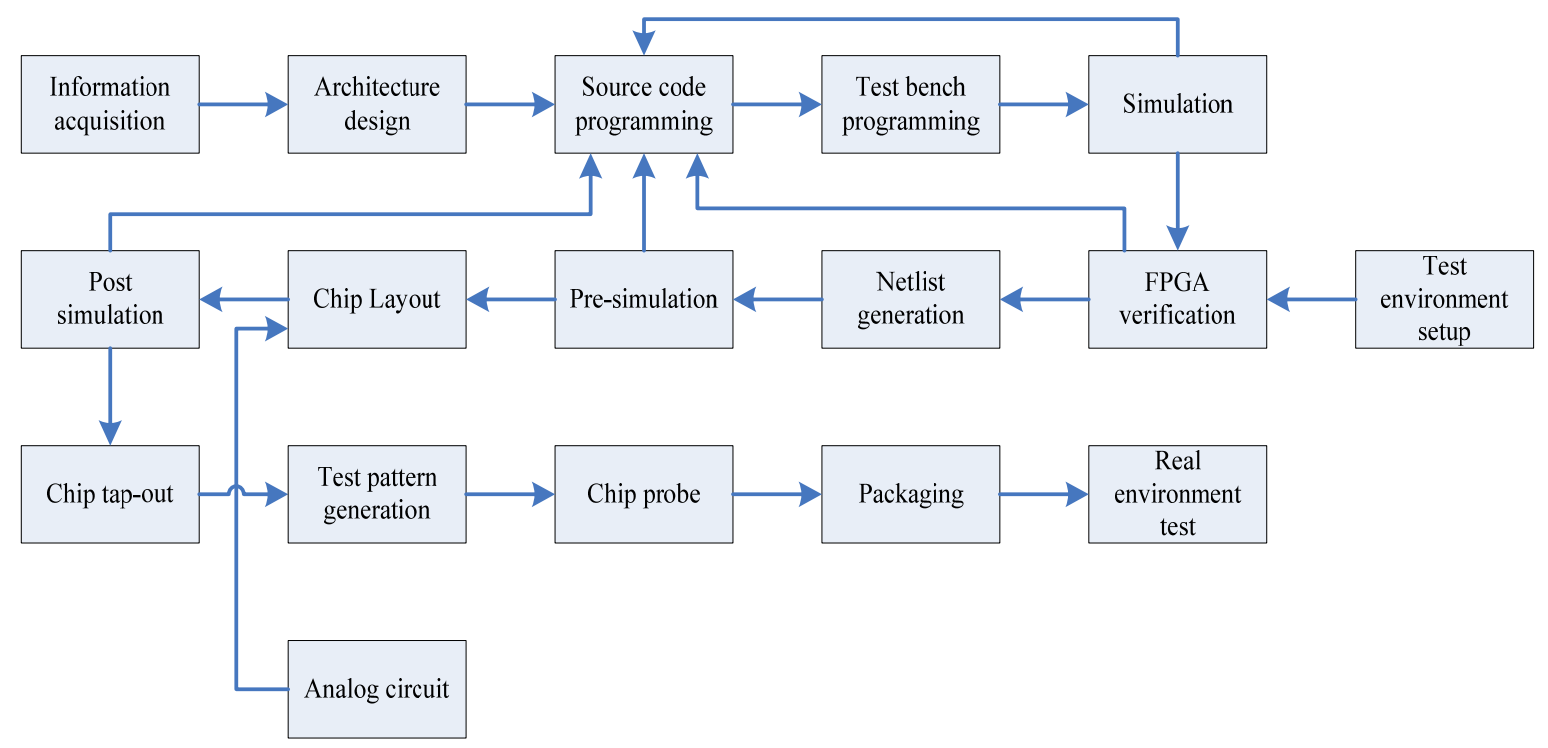

Figure 1. IC design flow chart. 
number of pixels which lie on the boundary between two regions. Edges are located in the areas with strong intensity contrasts. There are many edge detection operators based on the gradient detection [1]. The Sobel operator is chosen to be used for the edge detection in this paper.

The Sobel operator is a $3 \times 3$ mask used to compute the gradient for the corresponding region. Figure 2 shows pixels of the aimed image region, the Sobel operator multiplies with the image pixels to find out the gradient at the point labelled p5.

Sobel operators are as follows:

$$
\begin{gathered}
G_{x}=\left[\begin{array}{lll}
-1 & 0 & 1 \\
-2 & 0 & 2 \\
-1 & 0 & 1
\end{array}\right] \times A \\
G_{y}=\left[\begin{array}{ccc}
-1 & -2 & -1 \\
0 & 0 & 0 \\
1 & 2 & 1
\end{array}\right] \times A
\end{gathered}
$$

As shown in the Equations (1) and (2), $A$ is the grayscale of the original images. The $G_{x}$ is the row gradient and the $G_{y}$ is the column gradient. An approach used frequently is to approximate the magnitude of the Sobel gradient by absolute values:

$$
\nabla f \approx\left|G_{x}\right|+\left|G_{y}\right|
$$

After generating the value of the Equation (3), the result is compared with a threshold, which sets the final value to either black or white. The result is sent back to the image point labelled p5 for one round calculation.

\section{System Architecture}

In this research, the Sobel operator is used to detect the edge of a $256 \times 256$ grayscale image. The Sobel operation is separated into two parts, one part is a Sobel core, the other part is the Sobel full scan. The Sobel core is a single calculation of the matrix, and the Sobel full scan is used to scan the full image frame. The Sobel operator design is optimized for the hardware implementation from the following aspects:

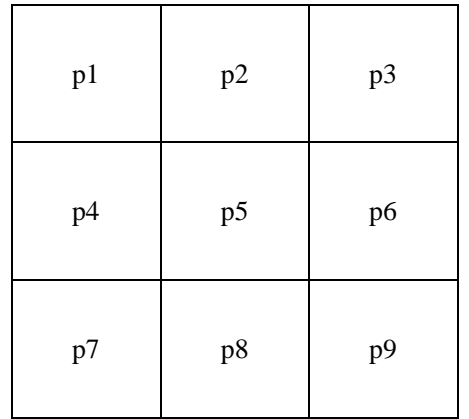

Figure 2. Image region for edge detection.
- The Sobel core needs higher frequency to finish the calculation in a pipeline design. In order to make the design run efficiently, the operation defines that one pixel is loaded to the Sobel core every clock cycle. After loading the input data, the Sobel operation can generate the output data in the following cycle. The Sobel core of this design needs two clock cycles to generate the result, so Sobel core part is connected to a clock, whose frequency is two times as the clock connected to Sobel full scan. Thus, the data can flow continuously without the latency.

- The other optimization is to put the generated data to the image pixel labelled p1 instead of $\mathrm{p} 5$ as shown in Figure 3. The design can have a higher efficiency by using this way. After the calculation of the single matrix. The Sobel mask will move to the next window, it will go through all the rows and columns in the image frame. If the generated results are sent back to $\mathrm{p} 5$, the Sobel mask must store the original pixel before the results are sent back. If designers want to avoid the influence of the results, they must use other storage devices to hold the results, which is a costly method. However, we store the results directly in the pixel labelled p1. The pixel labelled p1 will not be used in the future, so it has no influence for the future calculation even if the data is modified. Moreover, the designer does not need additional storage devices to keep results.

The designed hardware must be tested under the test environment, which is a system verification platform. The platform can be used to test the functionality of the design and it includes the following components:

- Test module design. The Design Under Test (DUT) module is connected to the platform as a slave.

- Verification module design. Functional simulation of the CPU is used to test the designed hardware. The memory controller and some external interface modules are designed and verified.

- Advanced Microcontroller Bus Architecture (AMBA) based protocol design. Advanced High-performance Bus (AHB) from the AMBA bus protocol is used for data transmission [7].

- Functional registers design. The hardware provides

\begin{tabular}{|c|c|c|c|c|c|c|}
\hline $\mathrm{p} 1$ & p2 & p3 & \multirow{3}{*}{$\begin{array}{c}\begin{array}{c}\text { Sobel } \\
\text { calculation }\end{array} \\
\square\end{array}$} & \multicolumn{2}{|c|}{$p_{1} / n_{2}$} & p3 \\
\hline $\mathrm{p} 4$ & p5 & p6 & & $\mathrm{p} 4$ & $\mathrm{p} 5$ & p6 \\
\hline p7 & p8 & p9 & & p7 & p8 & p9 \\
\hline
\end{tabular}
configuration registers for the software designer to design the corresponding software.

Figure 3. Optimized Sobel scheme. 
Figure 4 is the system verification platform. The verification platform includes the CPU, the memory controller, the DUT, and other interfaces. The platform uses AHB as the communication bus. Each DUT must have the slave interface and the master interface for communication through the AHB bus. As the central processing unit of the system, the CPU has only the master interface to send command. As the data storage devices, memories are viewed as slaves. Thus, they only have the slave interfaces.

The master interface is used to send commands to the slave interfaces and receive the data or responses from the slave interfaces. In the system design, the CPU will initial the command to the DUT, which is the Sobel edge detection module in this project, through the DUT's slave interface. The command is used to configure the functional registers of the designed hardware intellectual property (IP). Once the designed hardware gets the command from the CPU, it extracts the information and takes further actions. The information includes the start and the stop of the DUT, the initial address of the memory or other peripherals, where the DUT can fetch data from, etc. Then, the DUT can fetch the data from memory or other peripherals through its master interface to the slave interface of the memory controller. After finishing processing, the DUT can send the results back to the memory or other peripherals if necessary.

\section{Experimental Results}

The Sobel edge detection design is divided into two parts: one is the single matrix calculation; the other is the full frame calculation. Because the single calculation needs time to process the data, the clock frequency of the internal single calculation is twice as the full frame calculation to make the whole data run as the pipeline. In the real case, each gate has its own timing constraint. One can only use the maximum of around six adders or subtractors together to generate the output data through combinational logics. Thus, the single matrix calculation

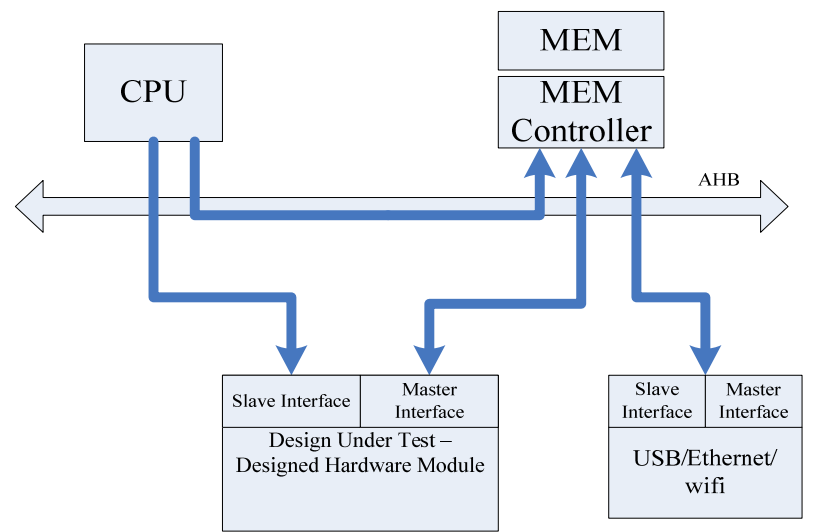

Figure 4. System verification platform. is separated into two RTL blocks. This can not only have the least register usage but the designed circuit can also run at a relatively higher frequency.

Compared to the single Sobel operator design in [2], they designed a single Sobel operator and mapped the design on Xilinx Spartan 3 XC3S50-5PQ208 board [8]. Their design can reach up to $190 \mathrm{MHz}$ frequency. In contrast, our design can only reach up to $156 \mathrm{MHz}$ on the same board. However, as for the resource costs, our design only occupies $10 \%$ of on-chip slices, while their design cost up to $16 \%$ of on-chip slices. In the System-on-a-Chip (SoC), on-chip resource costs are key factors which have great impacts on the design. The less of the resource costs, the lower of the power consumption. If a $256 \times 256$ frame needs to be processed, our design can ideally consume 0.41 ms to finish, while their design can ideally consume $0.34 \mathrm{~ms}$ to finish. This is still an acceptable time latency in the remote control system, especially consider the saved on-chip resources.

In another design from V. Sanduja and R. Patial [6], a $20 \times 40$ picture was processed by the Sobel edge detection design. Their design used Xilinx Virtex 4 FPGA board. The device was XC4VLX200, and the package was FF1513. Even they got the accurate result for each pixel, the design cost too much on-chip resource. Table 1 shows the device utilization comparison. It is shown that our design uses much less resources than their design. One advantage of our design is using a single RAM to store the data, after pixels are processed by the single matrix calculation. The processed result is sent back to the position labelled p1 instead of p5. This optimization method can save a large amount of storage space and the processed picture is usable for further steps in our SoC design. The other advantage is that our design does not process the rightmost two columns and lowest two rows of the picture. This can save the processing time when the data set is huge enough. Moreover, the omitted pixels have little influence to the final results.

For the verification platform, the CPU is instantiated as a functional module, and it sends command registers to the Sobel edge detection operator. The image frame used for the experiment is a $256 \times 256$ grayscale picture, so there are 65,536 positions in the memory. The Sobel edge detection operator extracts the information from the command registers, so that the operator knows when to

Table 1. Device utilization comparison.

\begin{tabular}{cccc}
\hline & $\begin{array}{c}\text { Number of } \\
\text { Occupied Slice }\end{array}$ & $\begin{array}{c}\text { Number of } \\
\text { Slice } \\
\text { Flip-Flops }\end{array}$ & $\begin{array}{c}\text { Number of } \\
\text { 4-Input LUTs }\end{array}$ \\
\hline $\begin{array}{c}\text { Design } \\
\text { in [6] }\end{array}$ & 1987 & 836 & 3901 \\
$\begin{array}{c}\text { Proposed } \\
\text { Design }\end{array}$ & 1144 & 128 & 1400 \\
\hline
\end{tabular}


write and read data. The Sobel edge detection operator also gets the information of where to fetch the data blocks. Then, the operator sends commands to the memory controller to fetch the data and do calculations. The system sends the results back to the memory after finishing the whole image frame calculation.

The AHB bus protocol is an industry standard, so that the platform can be used for other DUTs in the future if properly configured. The transmission on the AHB bus is 32 bit. The responses of the slaves are set to okay for easy use to ensure the communication is good for this design.
Figure 5 is the block design of the system. The system uses two synchronized clocks and one global reset signal. The Sobel core block is embedded in the Sobel_fullscan block. Two signals, which are do_fullscan and fullscan_ done, are reserved for future usage. If there are more than one DUTs in the system, an arbiter will be used to accommodate different DUTs based on the AHB protocol. The Sobel operator communicates with the off-chip memory through the ddr_controller. The Sobel core IP is integrated into the sobel_fullscan IP. Figure 6 is the simulation results from Mentor Graphic Modelsim [9]. By employing the pipeline technique, the DUT runs

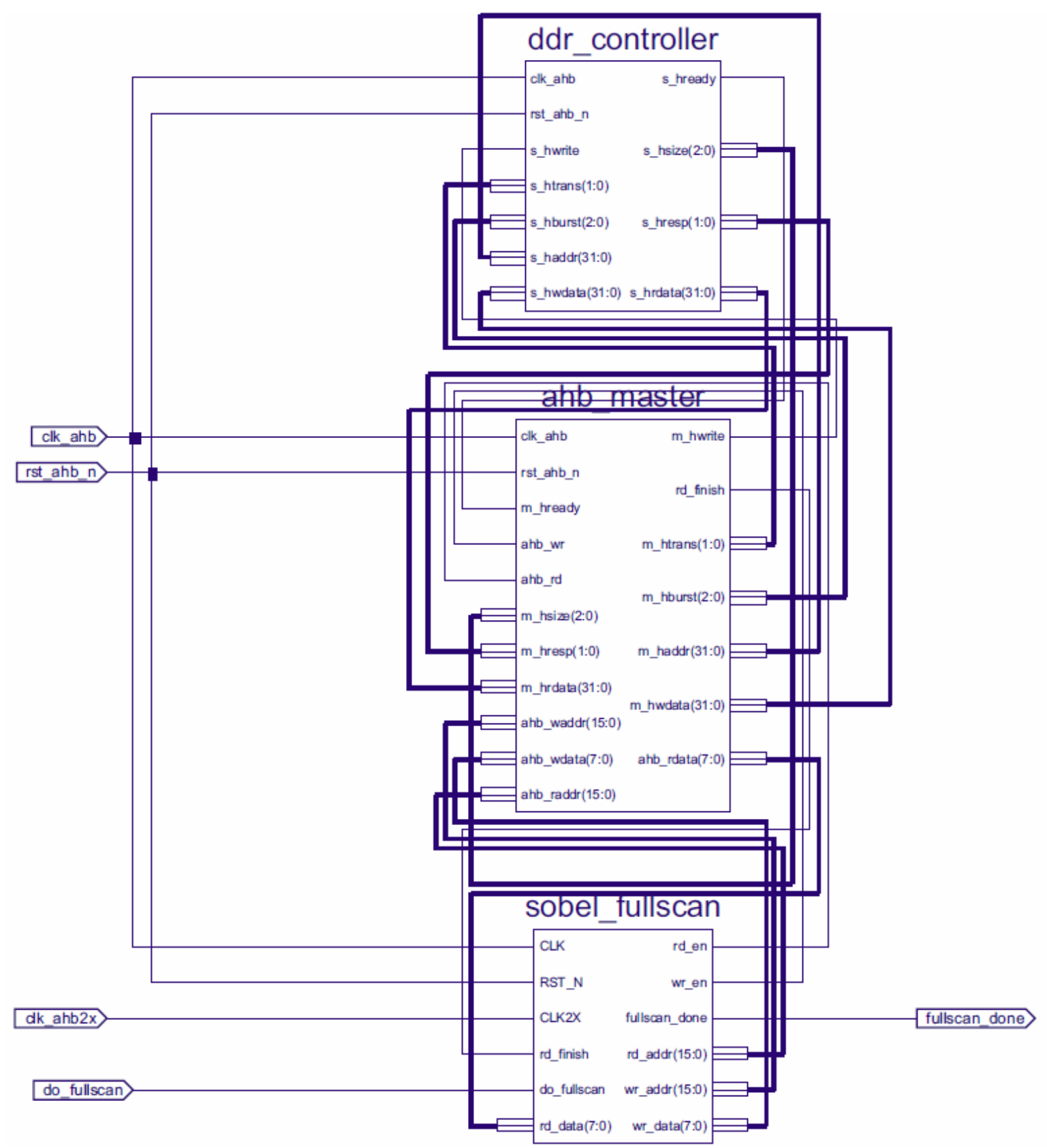

Figure 5. Schematic of Sobel operator with AHB bus. 

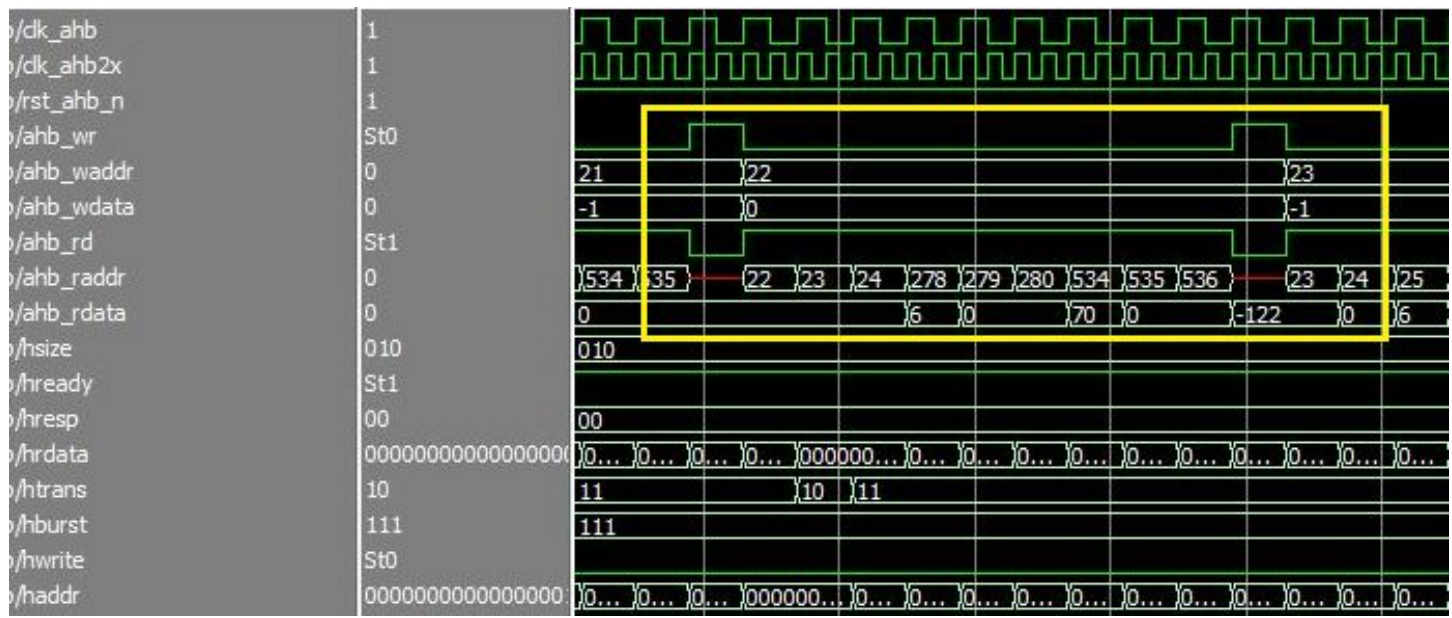

Figure 6. Simulation results with system bus.

smoothly on the verification platform. The edge detection part consumes nine clock cycles to fetch the desired pixels, then, the result can be generated in the tenth clock cycle. Thus, the design can not only have a shorter execution time but also few resource costs.

\section{Conclusions}

This paper introduces a system verification platform of hardware optimization based on the edge detection algorithm. The Sobel edge detection operator is designed and verified on the verification platform. The IC design flow is provided to make sure the designed chip is a good one. The Sobel edge detection operator is one of the most commonly used methods in remote controller design. In this design, the Sobel operator is separated into two parts in order to process the data more efficiently and consume fewer resources. Experimental results show that the designed Sobel edge detection operator can save $6 \%$ of on-chip resources for the Sobel core calculation and $42 \%$ for the whole frame calculation.

The system verification platform is set up to verify the designed hardware. The verification platform is composed of the functional CPU module, the DUT module, the memory module and other peripherals. These modules communicate with each other through the AHB bus protocol. It is proved that the designed Sobel operator works efficiently on this verification platform. The designed Sobel operator can be processed to the next stage to make an IC chip. The verification platform can be further used to verify other designed hardware.

\section{REFERENCES}

[1] R. C. Gonzalez and R. E. Woods, "Digital Image Proc- essing,” 2nd Edition, Prentice Hall, Upper Saddle River, 2001.

[2] S. Halder, D. Bhattacharjee, et al., "A Fast FPGA Based Architecture for Sobel Edge Detection,” Progress in VLSI Design and Test, Lecture Notes in Computer Science, Vol. 7373, 2012, pp. 300-306.

[3] T. A. Abbasi and M. U. Abbasi, "A Novel FPGA-Based Architecture for Sobel Edge Detection Operator," International Journal of Electronics, Vol. 94, No. 9, 2007, pp. 889-896. doi:10.1080/00207210701685253

[4] C. Pradabpet, N. Ravinu, et al., "An Efficient Filter Structure for Multiplierless Sobel Edge Detection,” Innovative Technologies in Intelligent Systems and Industrial Applications, 25-26 July 2009, pp. 40-44.

[5] Z. E. M. Osman, F. A. Hussin, et al., "Hardware Implementation of an Optimized Processor Architecture for Sobel Image Edge Detection Operator,” 2010 International Conference on Intelligent and Advanced Systems, 15-17 June 2010, pp. 1-4.

[6] V. Sanduja and R. Patial, "Sobel Edge Detection Using Parallel Architecture Based on FPGA," International Journal of Applied Information Systems, Vol. 4, No. 4, 2012, pp. 20-24.

[7] AMBA Specifications.

http://www.arm.com/products/system-ip/amba/amba-ope n-specifications.php

[8] Xilinx Incorporated. http://www.xilinx.com

[9] MentorGraphics Modelsim. http://www.model.com 